\title{
VOLTAGE-CURRENT CHARACTERISTICS OF A pn-DIODE FROM A DRIFT-DIFFUSION MODEL WITH NONLINEAR DIFFUSION
}

\author{
BY \\ ANSGAR JÜNGEL (Fachbereich Mathematik, TU Berlin, Germany) \\ AND \\ CHRISTIAN SCHMEISER (Institut für Angewandte \\ und Numerische Mathematik, TU Wien, Austria)
}

\begin{abstract}
A drift-diffusion model with density-dependent diffusion coefficients for the flow of electrons and holes in a semiconductor crystal is considered. It contains a new class of models for recombination-generation effects as well as boundary conditions modelling Ohmic contacts. Existence of steady-state solutions is proven. For a planar $p n$-diode the qualitative properties of steady-state solutions in dependence on the applied voltage is examined and, in particular, voltage-current characteristics are discussed.
\end{abstract}

1. Introduction. We consider a drift-diffusion model for the flow of electrons and holes in a semiconductor crystal with density-dependent diffusion coefficient. In particular, the situation is studied when the diffusion coefficient tends to zero as the density vanishes. Degenerate parabolic equations of this kind are used as models for the flow in porous media. It is well known that they allow for regions with positive densities to coexist with zero-density regions, henceforth termed vacuum regions. Recently it has been established that vacuum regions, i.e., regions depleted of either electrons or holes, are also predicted by the semiconductor drift-diffusion model with nonlinear diffusion [1], [4]. From a physical point of view this is not unreasonable since it is a basic fact that in semiconductor devices regions with very low carrier densities exist. This property of the model is responsible for one of the main results of this work: For the simplest semiconductor device, the $p n$-diode, in the absence of recombination-generation effects, the model predicts an idealized voltage-current characteristic. Its main property is that the current vanishes for applied biases below a threshold voltage which can be computed explicitly. In terms of semiconductor physics, nonvanishing current flow is only predicted in high-injection situations.

The paper is organized as follows. In Sec. 2 the model is presented. It consists of a steady-state version of the by now well-known drift-diffusion equations with nonlinear

Received December 29, 1994.

1991 Mathematics Subject Classification. Primary 78A55, 35R35.

Key words and phrases. Semiconductors, drift-diffusion model, degenerate elliptic equations. 
diffusion for electrons and holes, coupled to the Poisson equation for the electrostatic potential. We propose mixed boundary conditions modeling insulating boundary segments as well as Ohmic contacts. Also a class of models for recombination-generation phenomena is proposed. These models are motivated by well-established corresponding models for the standard drift-diffusion equations (with constant diffusivity, see [2]). By an appropriate scaling, the equations are made dimensionless.

In Sec. 3 existence of solutions is proven by regularization similar to [3]. The existence proof of that work had to be modified for our purposes since in [3] no recombinationgeneration effects have been considered, and the boundary data for the densities have been assumed to be bounded away from zero whereas we allow for the occurrence of a vacuum at the boundary.

In Sec. 4 we consider a one-dimensional model for a $p n$-diode. For the case of vanishing recombination-generation we obtain rather explicit information about solutions. In particular, the current through the device is shown to be zero for applied biases below a threshold voltage. The behaviour of the characteristic close to this threshold voltage is analyzed. We also obtain results on the monotonicity of the vacuum regions in terms of the applied bias. For the case of nonvanishing recombination-generation effects vacuum regions also appear. However, it has to be expected that the current vanishes only in thermal equilibrium, i.e., for zero applied bias.

2. The Model-Scaling. We consider the following drift-diffusion model for the electron and hole current densities:

$$
\mathbf{J}_{n}=q \mu_{n}\left(\frac{1}{q} \nabla r_{n}-n \nabla V\right), \quad \mathbf{J}_{p}=q \mu_{p}\left(-\frac{1}{q} \nabla r_{p}-p \nabla V\right),
$$

where $\mathbf{J}_{n}, \mu_{n}, r_{n}$, and $n$ denote the current density, the mobility, the pressure and, respectively, the density of the electrons. $\mathbf{J}_{p}, \mu_{p}, r_{p}$, and $p$ are the corresponding quantities for holes, $q$ is the elementary charge and $V$ the electrostatic potential. The steady-state continuity equations for electrons and holes are given by

$$
\nabla \cdot \mathbf{J}_{n}=-\nabla \cdot \mathbf{J}_{p}=R
$$

with the recombination-generation rate $R$. The electrostatic potential is determined from the Poisson equation

$$
\varepsilon_{s} \Delta V=q(n-p-C(x))
$$

with the semiconductor permittivity $\varepsilon_{s}$ and the prescribed doping profile $C(x)$ with $x \in \Omega \subset \mathbb{R}^{m}, m=1,2$, or 3 . Here $\Omega$ denotes the semiconductor domain.

The model is completed by constitutive laws for the pressures. We assume the pressures to be given as functions of the densities in the form

$$
r_{n}=k T n_{0} \tilde{r}\left(n / n_{0}\right), \quad r_{p}=k T n_{0} \tilde{r}\left(p / n_{0}\right)
$$

with the Boltzmann constant $k$, the lattice temperature $T$, and an increasing dimensionless function $\tilde{r}$ with $\tilde{r}(1)=1$. Then $n_{0}$ is the value of the density where the carrier 
temperature is equal to the lattice temperature. This parameter does not appear in the standard drift-diffusion model where $\tilde{r}$ is the identity. The diffusion current densities can be written in the form

$$
\mu_{n} \nabla r_{n}=q D_{n} \nabla n, \quad-\mu_{p} \nabla r_{p}=-q D_{p} \nabla p
$$

with the diffusivities

$$
D_{n}=\mu_{n} U_{T} \tilde{r}^{\prime}\left(n / n_{0}\right), \quad D_{p}=\mu_{p} U_{T} \tilde{r}^{\prime}\left(p / n_{0}\right),
$$

where the thermal voltage is given by $U_{T}=k T / q$. In the case of the standard driftdiffusion model the equations (2.3) are reduced to the so-called Einstein relations (see, e.g., [2]). As long as the diffusivities are positive, elliptic equations for the densities are obtained by substituting the current relations (2.1) in the continuity equations (2.2). Here, however, we are interested in the degenerate case where the diffusivities vanish for zero densities. In particular, the function $\tilde{r}$ determining the pressures will be assumed to be of the form

$$
\tilde{r}(\rho)=\rho^{\gamma}, \quad \gamma>1 .
$$

In the language of gas dynamics this means that we assume isentropic flow for the electron gas and the hole gas with the ratio of specific heats $\gamma$.

Before specifying the boundary conditions and the model for the recombinationgeneration rate, we introduce a scaling. With the reference length $L$ (e.g., the diameter of $\Omega$ ), the reference voltage $U_{T}$, the reference density $\widetilde{C}$ (typical for the doping profile), the reference mobility $\tilde{\mu}$, and the reference current density $q \tilde{\mu} \widetilde{C} U_{T} / L$ the dimensionless current relations read

$$
\mathbf{J}_{n}=\mu_{n}(\nabla r(n)-n \nabla V), \quad \mathbf{J}_{p}=\mu_{p}(-\nabla r(p)-p \nabla V)
$$

with $r(\rho)=\tilde{r}\left(\rho \tilde{C} / n_{0}\right)$. The scaled continuity equations are

$$
\nabla \cdot \mathbf{J}_{n}=-\nabla \cdot \mathbf{J}_{p}=R
$$

and the Poisson equation reads

$$
\lambda^{2} \Delta V=n-p-C
$$

after scaling, where the dimensionless parameter

$$
\lambda=\frac{1}{L} \sqrt{\frac{\varepsilon_{s} U_{T}}{q \widetilde{C}}}
$$

is the scaled Debye length. In the above equations the dimensionless variables and parameters are denoted by the same symbols as their dimensional counterparts.

The derivation of the boundary conditions and the recombination-generation model is based on a discussion of the thermal equilibrium state. First, we notice that the current relations can be formally rewritten as

$$
\mathbf{J}_{n}=\mu_{n} n \nabla(h(n)-V), \quad \mathbf{J}_{p}=-\mu_{p} p \nabla(h(p)+V),
$$


with

$$
h(\rho)=\int_{1}^{\rho} \frac{r^{\prime}(s)}{s} d s .
$$

Thermal equilibrium is a state with vanishing current densities. Therefore, in thermal equilibrium, we have on the one hand that either $n=0$ or $h(n)-V$ is constant and, on the other hand, that either $p=0$ or $h(p)+V$ is constant. Unfortunately, this information is not sufficient to specify the thermal equilibrium state completely. We shall see in Sec. 4 that different states with vanishing current densities can exist. As a resolution of this difficulty we adopt the view introduced in [3] and also used in the existence proof of the following section. There the model with nonlinear diffusion is considered as a limiting case of a regularized model where the diffusion coefficients are bounded away from zero. This regularization can be achieved in different ways. As in [4] we replace the pressure function by

$$
r^{\varepsilon}(\rho)= \begin{cases}r(\varepsilon) \rho / \varepsilon, & \rho \leq \varepsilon \\ r(\rho), & \rho \geq \varepsilon\end{cases}
$$

The function

$$
h^{\varepsilon}(\rho)=\int_{1}^{\rho} \frac{r^{\varepsilon \prime}(s)}{s} d s
$$

is defined accordingly. The function $h^{\varepsilon}$ satisfies $h^{\varepsilon}(0+)=-\infty$ for $\varepsilon>0$, whereas $h(0+)>-\infty$ holds. It is well known [3] that for the regularized model, vacuum does not occur. Therefore, thermal equilibrium is characterized by the requirement that $h^{\varepsilon}(n)-V$ ad $h^{\varepsilon}(p)+V$ are constant. Eliminating the potential, we obtain that $h^{\varepsilon}(n)+h^{\varepsilon}(p)$ is constant. As for the standard drift-diffusion model we write the value of this constant in terms of the intrinsic density $n_{i}$, i.e., the equilibrium density of electrons and holes in an undoped piece of semiconductor:

$$
h^{\varepsilon}(n)+h^{\varepsilon}(p)=2 h^{\varepsilon}\left(n_{i}\right) .
$$

This is the equation of a curve in the $(n, p)$-plane having the axes as asymptotes. It is easy to see that the limit of this curve as $\varepsilon \rightarrow 0$ is the set of all points satisfying

$$
n p\left(h(n)+h(p)-2 h\left(n_{i}\right)\right)=0, \quad h(n)+h(p)-2 h\left(n_{i}\right) \geq 0 .
$$

This equilibrium curve corresponds to the equilibrium condition $n p=n_{i}^{2}$ for the standard drift-diffusion model. To describe the equilibrium state further, we turn back to the regularized model. We have to specify the constant values of $h^{\varepsilon}(n)-V$ and of $h^{\varepsilon}(p)+V$ such that their sum is $2 h^{\varepsilon}\left(n_{i}\right)$. This corresponds to choosing a reference value for the potential and we set

$$
h^{\varepsilon}(n)-V=h^{\varepsilon}\left(n_{i}\right), \quad h^{\varepsilon}(p)+V=h^{\varepsilon}\left(n_{i}\right)
$$

Obviously, $h^{\varepsilon}$ is strictly monotone and maps $(0, \infty)$ onto $\mathbb{R}$. We denote its inverse by $g^{\varepsilon}$ and rewrite the above equations as

$$
n=g^{\varepsilon}\left(V+h^{\varepsilon}\left(n_{i}\right)\right), \quad p=g^{\varepsilon}\left(-V+h^{\varepsilon}\left(n_{i}\right)\right) \text {. }
$$


In the limit $\varepsilon \rightarrow 0$ we obtain

$$
n=g\left(V+h\left(n_{i}\right)\right), \quad p=g\left(-V+h\left(n_{i}\right)\right)
$$

where the function $g$ vanishes for arguments smaller than $h(0+)$ and coincides with the inverse of $h$ for arguments greater than $h(0+)$.

Now we are in the position to formulate boundary conditions for Ohmic contacts. As for the standard drift-diffusion model we assume that the carrier densities at Ohmic contacts are in thermal equilibrium and that the space charge density $-n+p+C$ vanishes. Therefore, the boundary values of the densities are the coordinates of the unique point $\left(n_{D}, p_{D}\right)$ of intersection of the equilibrium curve and the line $n-p-C=0$. Depending on the value of the doping density at the boundary, different cases can occur. While for $|C|<n_{\text {vac }}$ with

$$
n_{\mathrm{vac}}=g\left(2 h\left(n_{i}\right)-h(0+)\right)
$$

both densities are positive, vacuum occurs at the boundary if $|C| \geq n_{\mathrm{vac}}$ holds. Then, for $C>0$ we have $n_{D}=C, p_{D}=0$ and for $C<0$ we have $n_{D}=0, p_{D}=-C$.

The values of the thermal equilibrium potential at Ohmic contacts are obtained by $\varepsilon \rightarrow 0$ in (2.13). However, we have to be careful since $h^{\varepsilon}$ only has a limit for positive arguments. Since always at least one of the two densities $n$ and $p$ is positive, the potential can be computed from the corresponding equation in (2.13). As for the standard driftdiffusion model we call these boundary values the built-in potential:

$$
V_{\mathrm{bi}}(C)= \begin{cases}h(C)-h\left(n_{i}\right), & C \geq n_{\mathrm{vac}} \\ h\left(n_{D}\right)-h\left(n_{i}\right)=h\left(n_{i}\right)-h\left(p_{D}\right), & |C|<n_{\mathrm{vac}} \\ h\left(n_{i}\right)-h(-C), & C \leq-n_{\mathrm{vac}}\end{cases}
$$

Note that the built-in potential can also be characterized as the unique solution of the equation

$$
g\left(V_{\mathrm{bi}}+h\left(n_{i}\right)\right)-g\left(h\left(n_{i}\right)-V_{\mathrm{bi}}\right)-C=0 .
$$

In a nonequilibrium situation, boundary values for the potential are obtained by adding the externally applied voltage at a particular Ohmic contact to $V_{\mathrm{bi}}(C)$.

For recombination-generation mechanisms we use a class of models compatible with the nonlinear drift-diffusion model in he sense that the recombination-generation term vanishes in thermal equilibrium:

$$
R(n, p)=Q(n, p) F\left(h(n)+h(p)-2 h\left(n_{i}\right)\right)
$$

with

$$
\begin{gathered}
F(0)=0, \quad F \text { increasing, } \quad Q(n, p) \geq 0 \\
Q(n, 0)=0 \text { for } n \geq n_{\mathrm{vac}}, \quad Q(0, p)=0 \text { for } p \geq n_{\mathrm{vac}} .
\end{gathered}
$$

3. Existence of steady-state solutions. In this section we consider the system (2.5), (2.6), (2.7) with the pressure law (2.4) and a recombination-generation term of 
the form (2.16), (2.17) in a domain $\Omega \subset \mathbb{R}^{d}, d=1,2$, or 3 . The boundary of $\Omega$ can be written as the disjoint union

$$
\partial \Omega=\partial \Omega_{N} \cup C_{1} \cup \cdots \cup C_{k}
$$

with the Ohmic contacts $C_{1}, \ldots, C_{k}$ and the union $\partial \Omega_{N}$ of the insulating (or artificial) boundary segments.

Denoting the applied voltages at the Ohmic contacts by $U_{1}, \ldots, U_{k}$, Dirichlet boundary data are determined by

$$
\left.\begin{array}{l}
n_{D} p_{D}\left(h\left(n_{D}\right)+h\left(p_{D}\right)-2 h\left(n_{i}\right)\right)=0, \\
h\left(n_{D}\right)+h\left(p_{D}\right)-2 h\left(n_{i}\right) \geq 0, \\
n_{D}-p_{D}-C=0, \\
V_{D}=V_{\mathrm{bi}}+U_{j},
\end{array}\right\} \quad \text { on } C_{j}, j=1, \ldots, k,
$$

where the built-in potential is defined by $(2.15)$. Then we have the boundary conditions

$$
\begin{aligned}
n=n_{D}, \quad p=p_{D}, \quad V=V_{D}, & \text { on } \partial \Omega_{D}=C_{1} \cup \cdots \cup C_{k}, \\
\mathbf{J}_{n} \cdot \boldsymbol{\nu}=\mathbf{J}_{p} \cdot \boldsymbol{\nu}=\nabla V \cdot \boldsymbol{\nu}=0, & \text { on } \partial \Omega_{N},
\end{aligned}
$$

where $\boldsymbol{\nu}$ denotes the outward unit normal along $\partial \Omega_{N}$.

For the results of this section we need the following technical assumptions:

(H1) $\Omega \subset \mathbb{R}^{d}$ is a bounded domain with $C^{0,1}$ boundary $\partial \Omega=\partial \Omega_{D} \cup \partial \Omega_{N}$ where $\partial \Omega_{D} \cap$ $\partial \Omega_{N}=\{\}, \partial \Omega_{N}$ is open in $\partial \Omega$, and the $(d-1)$-dimensional Lebesgue measure of $\partial \Omega_{D}$ is positive.

(H2) $C \in L^{\infty}(\Omega)$. The Dirichlet boundary data for the potential determined by (3.1) have an extension to $\Omega$ satisfying $V_{D} \in L^{\infty}(\Omega) \cap H^{1}(\Omega)$. This assumption is satisfied if $C$ is sufficiently smooth near the contacts.

(H3) The functions $Q$ and $F$, determining the recombination-generation rate, are smooth. Now we can formulate the main result of this section.

THEOREM 3.1. Let the pressure be given by (2.4) and the recombination-generation rate by $(2.16),(2.17)$. Let the assumptions (H1)-(H3) hold. Then there exists a weak solution $(n, p, V) \in\left(L^{\infty}(\Omega)\right)^{3}$ with $n, p \geq 0$ and $(r(n), r(p), V) \in\left(H^{1}(\Omega)\right)^{3}$ of the problem (2.5)$(2.7),(3.2)$.

Proof. The proof is similar to that of Theorem 3.1 in [3]. The main idea is to consider a regularized version of the problem where the pressure law (2.4) is replaced by (2.10). Accordingly, the functions $h$ and $g$ are replaced by $h^{\varepsilon}$ (see (2.11)) and its inverse $g^{\varepsilon}$, respectively. In the regularized problem (with the unknowns $n^{\varepsilon}, p^{\varepsilon}, V^{\varepsilon}$ ) we introduce the new variables

$$
\varphi_{n}^{\varepsilon}=V^{\varepsilon}-h^{\varepsilon}\left(n^{\varepsilon}\right)+h^{\varepsilon}\left(n_{i}\right), \quad \varphi_{p}^{\varepsilon}=V^{\varepsilon}+h^{\varepsilon}\left(p^{\varepsilon}\right)-h^{\varepsilon}\left(n_{i}\right) .
$$

In analogy to the standard drift-diffusion model we shall call $\varphi_{n}^{\varepsilon}$ and $\varphi_{p}^{\varepsilon}$ the quasi-Fermi potentials.

A simple computation shows that in terms of the quasi-Fermi potentials we obtain the current relations

$$
\mathbf{J}_{n}^{\varepsilon}=-\mu_{n} n^{\varepsilon} \nabla \varphi_{n}^{\varepsilon}, \quad \mathbf{J}_{p}^{\varepsilon}=-\mu_{p} p^{\varepsilon} \nabla \varphi_{p}^{\varepsilon}
$$


and the continuity equations

$$
\nabla \cdot \mathbf{J}_{n}^{\varepsilon}=-\nabla \cdot \mathbf{J}_{p}^{\varepsilon}=R^{\varepsilon}=Q\left(n^{\varepsilon}, p^{\varepsilon}\right) F\left(\varphi_{p}^{\varepsilon}-\varphi_{n}^{\varepsilon}\right),
$$

with the densities being given by

$$
n^{\varepsilon}=g^{\varepsilon}\left(V^{\varepsilon}-\varphi_{n}^{\varepsilon}+h^{\varepsilon}\left(n_{i}\right)\right), \quad p^{\varepsilon}=g^{\varepsilon}\left(\varphi_{p}^{\varepsilon}-V^{\varepsilon}+h^{\varepsilon}\left(n_{i}\right)\right) .
$$

The quasi-Fermi potentials satisfy the boundary conditions

$$
\varphi_{n}^{\varepsilon}=\varphi_{p}^{\varepsilon}=U_{D} \quad \text { on } \partial \Omega_{D}, \quad \nabla \varphi_{n}^{\varepsilon} \cdot \boldsymbol{\nu}=\nabla \varphi_{p}^{\varepsilon} \cdot \boldsymbol{\nu}=0 \quad \text { on } \partial \Omega_{N}
$$

where $U_{D}$ is an $H^{1}(\Omega)$-extension of the applied voltages:

$$
U_{D}=U_{j} \quad \text { on } C_{j}, j=1, \ldots, k \text {. }
$$

Now we construct a fixed-point map for the regularized problem. Given approximations $\varphi_{n}, \varphi_{p}$ for the quasi-Fermi potential with

$$
\varphi_{n}, \varphi_{p} \in \mathcal{S}=\left\{\varphi \in H^{1}(\Omega): \underline{U} \leq \varphi \leq \bar{U}\right\}
$$

with

$$
\underline{U}=\min _{1 \leq j \leq k} U_{j}, \quad \bar{U}=\max _{1 \leq j \leq k} U_{j},
$$

we compute an approximation $V$ of the potential by solving

$$
\begin{gathered}
\lambda^{2} \Delta V=g^{\varepsilon}\left(V-\varphi_{n}+h^{\varepsilon}\left(n_{i}\right)\right)-g^{\varepsilon}\left(\varphi_{p}-V+h^{\varepsilon}\left(n_{i}\right)\right)-C, \\
V=V_{D} \quad \text { on } \partial \Omega_{D}, \quad \nabla V \cdot \boldsymbol{\nu}=0 \quad \text { on } \partial \Omega_{N} .
\end{gathered}
$$

By the monotonicity of $g^{\varepsilon}$ it follows from standard results that this problem has a unique solution in $H^{1}(\Omega) \cap L^{\infty}(\Omega)$ satisfying

$$
V_{\mathrm{bi}}^{\varepsilon}(\underline{C})+\underline{U} \leq V \leq V_{\mathrm{bi}}^{\varepsilon}(\bar{C})+\bar{U},
$$

with $\underline{C}=\inf _{\Omega} C, \bar{C}=\sup _{\Omega} C$ and the regularized built-in potential $V_{\mathrm{bi}}^{\varepsilon}(C)$ defined by

$$
g^{\varepsilon}\left(V_{\mathrm{bi}}^{\varepsilon}+h^{\varepsilon}\left(n_{i}\right)\right)-g^{\varepsilon}\left(h^{\varepsilon}\left(n_{i}\right)-V_{\mathrm{bi}}^{\varepsilon}\right)-C=0 .
$$

Now approximations for the densities are computed from

$$
n=g^{\varepsilon}\left(V-\varphi_{n}+h^{\varepsilon}\left(n_{i}\right)\right), \quad p=g^{\varepsilon}\left(\varphi_{p}-V+h^{\varepsilon}\left(n_{i}\right)\right) \text {. }
$$

Note that for fixed positive $\varepsilon$ these densities are bounded away from zero uniformly in $\Omega$. Finally, the problems

$$
\begin{gathered}
\nabla \cdot\left(\mu_{n} n \nabla \Phi_{n}\right)=-Q(n, p) F\left(\varphi_{p}-\Phi_{n}\right), \\
\Phi_{n}=U_{D} \quad \text { on } \partial \Omega_{D}, \quad \nabla \Phi_{n} \cdot \nu=0 \quad \text { on } \partial \Omega_{N},
\end{gathered}
$$


and

$$
\begin{gathered}
\nabla \cdot\left(\mu_{p} p \nabla \Phi_{p}\right)=Q(n, p) F\left(\Phi_{p}-\Phi_{n}\right), \\
\Phi_{p}=U_{D} \quad \text { on } \partial \Omega_{D}, \quad \nabla \Phi_{p} \cdot \nu=0 \quad \text { on } \partial \Omega_{N},
\end{gathered}
$$

are solved to obtain new approximations $\left(\Phi_{n}, \Phi_{p}\right)=\mathcal{T}\left(\varphi_{n}, \varphi_{p}\right)$. Again, the unique solvability of the problems and $\Phi_{n}, \Phi_{p} \in \mathcal{S}$ is immediate. (The properties (2.17) of the recombination-generation model admit the application of the maximum principle.)

Thus, $\mathcal{T}$ maps the convex, closed subset $\mathcal{S}^{2}$ of $\left(L^{2}(\Omega)\right)^{2}$ into itself. Standard arguments show that $\mathcal{T}$ is continuous and that $\mathcal{T}\left(\mathcal{S}^{2}\right)$ is precompact in $\left(L^{2}(\Omega)\right)^{2}$. Schauder's fixedpoint theorem implies the existence of a weak solution of the regularized problem for every positive $\varepsilon$.

From the above it is obvious that the solution of the regularized problem satisfies the estimates

$$
\begin{gathered}
V_{\mathrm{bi}}^{\varepsilon}(\underline{C})+\underline{U} \leq V \leq V_{\mathrm{bi}}^{\varepsilon}(\bar{C})+\bar{U} \\
g^{\varepsilon}\left(\underline{U}-\bar{U}+V_{\mathrm{bi}}^{\varepsilon}(\underline{C})+h^{\varepsilon}\left(n_{i}\right)\right) \leq n \leq g^{\varepsilon}\left(\bar{U}-\underline{U}+V_{\mathrm{bi}}^{\varepsilon}(\bar{C})+h^{\varepsilon}\left(n_{i}\right)\right), \\
g^{\varepsilon}\left(\underline{U}-\bar{U}-V_{\mathrm{bi}}^{\varepsilon}(\bar{C})+h^{\varepsilon}\left(n_{i}\right)\right) \leq p \leq g^{\varepsilon}\left(\bar{U}-\underline{U}-V_{\mathrm{bi}}^{\varepsilon}(\underline{C})+h^{\varepsilon}\left(n_{i}\right)\right) .
\end{gathered}
$$

Since the bounds above have finite limits as $\varepsilon \rightarrow 0$, it follows that $V, n$, and $p$ are uniformly bounded in $L^{\infty}(\Omega)$ as $\varepsilon \rightarrow 0$. Note that in general the lower bounds for the densities tend to zero as $\varepsilon \rightarrow 0$. Therefore the limits of the densities are not necessarily bounded away from zero and vacuum regions can occur.

From this point on the argument is as in the proof of Theorem 3.1 of [4]. It consists of uniform estimates for $V, r(n)$ and $r(p)$ in $H^{1}(\Omega)$, and of a justification of the limit $\varepsilon \rightarrow 0$ in the regularized problem.

4. Voltage-current characteristics of one-dimensional pn-diodes. In this section a one-dimensional version of the model $(2.5),(2.6),(2.7)$ with vanishing recombination-generation rate is considered. The doping profile models a $p n$-junction. Towards the end of this section the effects of nonvanishing recombination-generation will be discussed.

For the following we slightly change the definition of the function $h$, as compared to (2.9), by using 0 instead of 1 as a lower bund in the integration:

$$
h(\rho)=\int_{0}^{\rho} \frac{r^{\prime}(s)}{s} d s .
$$

Accordingly, $g$ will from now on denote the inverse of $h$ for positive arguments and the zero function for nonpositive arguments. This change simplifies the notation, since now $h(0+)=0$ holds.

The one-dimensional model consists of the current relations

$$
J_{n}=\mu_{n}\left(r(n)^{\prime}-n V^{\prime}\right), \quad J_{p}=\mu_{p}\left(-r(p)^{\prime}-p V^{\prime}\right)
$$

the continuity equations

$$
J_{n}^{\prime}=J_{p}^{\prime}=0
$$


and the Poisson equation

$$
\lambda^{2} V^{\prime \prime}=n-p-C,
$$

for $0<x<1$. For the doping profile we assume

$$
\begin{gathered}
C(0)>g\left(2 h\left(n_{i}\right)\right), \quad C(1)<-g\left(2 h\left(n_{i}\right)\right), \\
C \text { is monotonically decreasing. }
\end{gathered}
$$

Thus, there is an $n$-region $(C>0)$ next to the left end and a $p$-region $(C<0)$ next to the right end of the device. These names are explained by the fact that in the $n$-region the positive background charge attracts negatively charged electrons, and in the $p$-region the negative background charge attracts positively charged holes.

The first two assumptions in (4.4) are always satisfied in practical applications. They simplify the boundary conditions. We assume Ohmic contacts at $x=0$ and $x=1$. Then the boundary conditions for the densities become

$$
n(0)=C(0), \quad p(0)=0, \quad n(1)=0, \quad p(1)=-C(1)
$$

(see (3.1)). For the potential we obtain from $(2.15),(3.1)$

$$
V(0)=h(C(0))-h\left(n_{i}\right), \quad V(1)=h\left(n_{i}\right)-h(-C(1))+U
$$

with the externally applied voltage $U$.

The total current is given by $J=J_{n}+J_{p}$. We are interested in the voltage-current characteristic, i.e., the dependence of $J$ on the applied voltage $U$.

As a first step we consider thermal equilibrium, i.e., $U=0$. From Sec. 2 it is clear that the equilibrium potential is the solution of the semilinear elliptic problem

$$
\begin{array}{r}
\lambda^{2} V_{e}^{\prime \prime}=g\left(V_{e}+h\left(n_{i}\right)\right)-g\left(-V_{e}+h\left(n_{i}\right)\right)-C, \\
V_{e}(0)=h(C(0))-h\left(n_{i}\right), \quad V_{e}(1)=h\left(n_{i}\right)-h(-C(1)),
\end{array}
$$

with the equilibrium densities being given by $n_{e}=g\left(V_{e}=h\left(n_{i}\right)\right)$ and $p_{e}=g\left(-V_{e}+h\left(n_{i}\right)\right)$. LEMMA 4.1. The problem (4.7) has a unique, strictly monotonically decreasing solution $V_{e} \in C^{1,1}([0,1])$. There are points $0<x_{p e}<x_{n e}<1$ such that

$$
n_{e}\left\{\begin{array} { l l } 
{ > 0 , } & { x < x _ { n e } , } \\
{ = 0 , } & { x \geq x _ { n e } , }
\end{array} \quad p _ { e } \left\{\begin{array}{ll}
=0, & x \leq x_{p e} \\
>0, & x>x_{p e}
\end{array}\right.\right.
$$

Proof. Problem (4.7) is a one-dimensional version of (3.3). Thus, existence and uniqueness again follow from standard results for semilinear elliptic equations. The Lipschitz continuity of the second derivative of $V_{e}$ is an immediate consequence of the Lipschitz continuity of $g$. Monotonicity follows from a maximum principle for the differentiated equation. Here the monotonicity of the doping profile is used and the fact that the solution takes its extremal values at the boundaries. Finally, $x_{p e}$ and $x_{n e}$ are the points where $V_{e}$ takes the values $h\left(n_{i}\right)$ and $-h\left(n_{i}\right)$. 
REMARK. Note that we did not prove that problem (4.7) is equivalent to (4.1)-(4.3), (4.5), (4.6) with $U=0$. It is obvious that the solution of (4.7) solves (4.1)-(4.3), (4.5), (4.6) with $U=0$. However, no uniqueness result for the latter problem is known. Uniqueness is guaranteed only if we consider the limiting procedure $\varepsilon \rightarrow 0$, as carried out in the preceding section, as part of the problem formulation.

Now we turn to the nonequilibrium case. For small enough applied voltages $U$ we try to construct solutions with the same qualitative behaviour as the equilibrium solution, i.e., with

$$
n\left\{\begin{array} { l l } 
{ > 0 , } & { x < x _ { n } , } \\
{ = 0 , } & { x \geq x _ { n } , }
\end{array} \quad p \left\{\begin{array}{ll}
=0, & x \leq x_{p}, \\
>0, & x>x_{p}
\end{array}\right.\right.
$$

where the points $x_{n}, x_{p} \in(0,1)$ are yet to be determined. Since vacuum occurs for both electrons and holes in intervals of positive length, $J_{n}=J_{p}=0$ holds. In the interval $\left[0, x_{n}\right)$ where $n$ is positive, the representation (2.8) of the electron current can be used, and we have

$$
0=J_{n}=\mu_{n} n(h(n)-V)^{\prime}
$$

implying

$$
h(n)-V=h\left(n_{i}\right) \quad \text { in }\left[0, x_{n}\right),
$$

where the value of the constant on the right-hand side is obtained from the boundary conditions (4.5), (4.6) at $x=0$. Analogously we get

$$
h(p)+V=h\left(n_{i}\right)+U \quad \text { in }\left(x_{p}, 1\right] .
$$

As the next step we compute $n$ and $p$ from (4.8) and (4.9), respectively, and assume

$$
n=g\left(V+h\left(n_{i}\right)\right), \quad p=g\left(-V+h\left(n_{i}\right)+U\right) \quad \text { in }[0,1]
$$

Note that it is an extension of (4.8) and (4.9) that we assume the validity of (4.10) on the whole interval $[0,1]$. This assumption will be justified, if the solution satisfies

$$
V(x)\left\{\begin{array} { l l } 
{ > - h ( n _ { i } ) , } & { x < x _ { n } , } \\
{ \leq - h ( n _ { i } ) , } & { x \geq x _ { n } , }
\end{array} \quad V ( x ) \left\{\begin{array}{ll}
\geq h\left(n_{i}\right)+U, & x \leq x_{p} \\
>h\left(n_{i}\right)+U, & x>x_{p}
\end{array}\right.\right.
$$

With (4.10) the problem can again be reduced to a semilinear elliptic equation:

$$
\begin{gathered}
\lambda^{2} V^{\prime \prime}=g\left(V+h\left(n_{i}\right)\right)-g\left(-V+h\left(n_{i}\right)+U\right)-C, \\
V(0)=h(C(0))-h\left(n_{i}\right), \quad V(1)=h\left(n_{i}\right)-h(-C(1))+U .
\end{gathered}
$$

From the boundary conditions it is clear that (4.11) can only be satisfied if

$$
U \leq U_{\mathrm{th}}
$$

holds, where the threshold voltage is defined by $U_{\mathrm{th}}=\min \left\{h(C(0))-2 h\left(n_{i}\right), h(-C(1))-\right.$ $\left.2 h\left(n_{i}\right)\right\}>0$. 
Lemma 4.2. Let (4.13) hold. Then the problem (4.12) has a unique, strictly monotonically decreasing solution $V \in C^{1,1}([0,1])$. There are unique points $x_{n}$ ad $x_{p}$ in $[0,1]$ with $V\left(x_{n}\right)=-h\left(n_{i}\right)$ and $V\left(x_{p}\right)=h\left(n_{i}\right)+U$. The vacuum regions shrink strictly monotonically in terms of $U$, i.e., $x_{n}$ is a strictly monotonically increasing and $x_{p}$ a strictly monotonically decreasing function of $U$.

Proof. The first part of the proof is as the proof of Lemma 4.1.

It remains to prove the monotonicity of $x_{n}(U)$ and $x_{p}(U)$. Let $U_{1}<U_{2} \leq U_{\text {th }}$ hold. Then we denote the solutions of (4.12) with $U=U_{1}$ and $U=U_{2}$ by $V_{1}$ and $V_{2}$, respectively. An easy calculation shows that $V_{2}$ is an upper solution of the problem for $V_{1}$, and, therefore, $V_{1}(x)<V_{2}(x)$ for $x \in(0,1)$ follows. This implies

$$
V_{1}\left(x_{n}\left(U_{1}\right)\right)=-h\left(n_{i}\right)=V_{2}\left(x_{n}\left(U_{2}\right)\right)>V_{1}\left(x_{n}\left(U_{2}\right)\right)
$$

Since $V_{1}$ is strictly monotonically decreasing, we obtain $x_{n}\left(U_{1}\right)<x_{n}\left(U_{2}\right)$.

Considering the problem for $V-U$, another comparison function argument shows $V_{1}-U_{1}>V_{2}-U_{2}$ in $(0,1)$ and, thus,

$$
V_{1}\left(x_{p}\left(U_{1}\right)\right)-U_{1}=h\left(n_{i}\right)=V_{2}\left(x_{p}\left(U_{2}\right)\right)-U_{2}<V_{1}\left(x_{p}\left(U_{2}\right)\right)-U_{1},
$$

implying $x_{p}\left(U_{1}\right)>x_{p}\left(U_{2}\right)$, again by the strict monotonicity of $V_{1}$.

All the solutions constructed so far have zero total current. Thus, for $U \leq U_{\mathrm{th}}$, the voltage-current characteristic is identical to the $U$-axis. The threshold voltage $U_{\text {th }}$ is the applied bias where either the electron vacuum set or the hole vacuum set shrinks to one point. For $U>U_{\text {th }}$ we expect a total current different from zero. In this situation, however, we are not able to obtain information about the solution as explicit as in the case of vanishing current. Figure 1 (see p. 718) contains numerically computed (see [2]) electron and hole densities for thermal equilibrium, a reverse bias $U<0$, a forward bias $0<U<U_{\mathrm{th}}$, and a forward bias $U>U_{\mathrm{th}}$.

In the following we shall carry out a formal asymptotic analysis of the characteristic for $U$ close to $U_{\mathrm{th}}$. In the model (2.4) for the pressure function we shall assume

$$
1<\gamma<2
$$

The parameter $\alpha=\frac{1}{\gamma-1}>1$ will be used.

For definiteness we assume $C(0)>-C(1)$ and, thus, $U_{\text {th }}=h(-C(1))-2 h\left(n_{i}\right)$. In this case we have $x_{n}\left(U_{\mathrm{th}}\right)=1$, i.e., the electron vacuum region shrinks to the right boundary point as the applied bias reaches the value of the threshold voltage. In this case $n>0$ holds in the interior of the interval, and we expect this to remain true also for larger biases. In the following it will be convenient to work with the electron quasiFermi potential $\varphi_{n}=V-h(n)+h\left(n_{i}\right)$ instead of the electron density. Electron and hole densities are then given by

$$
n=g\left(V-\varphi_{n}+h\left(n_{i}\right)\right), \quad p=g\left(-V+h\left(n_{i}\right)+U\right)
$$

for $U$ larger than, but close to $U_{\text {th }}$. The expression for the hole density is the same as above since we expect a hole vacuum region of finite length. Note that the problem 
(a)

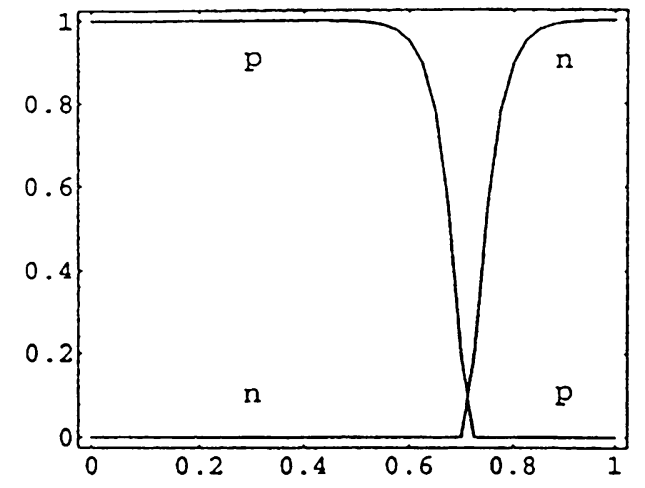

(c)

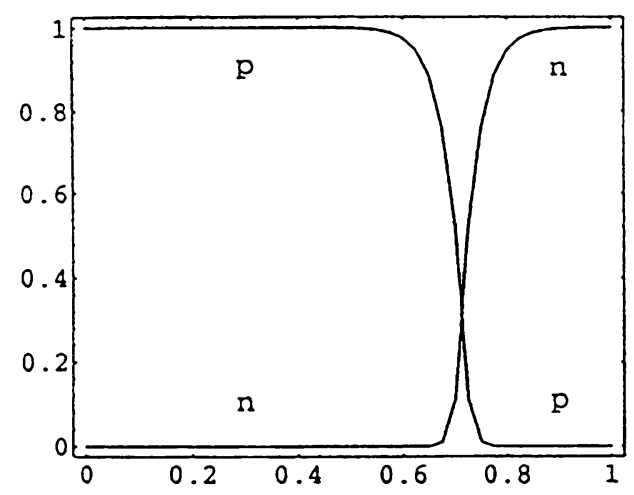

(b)

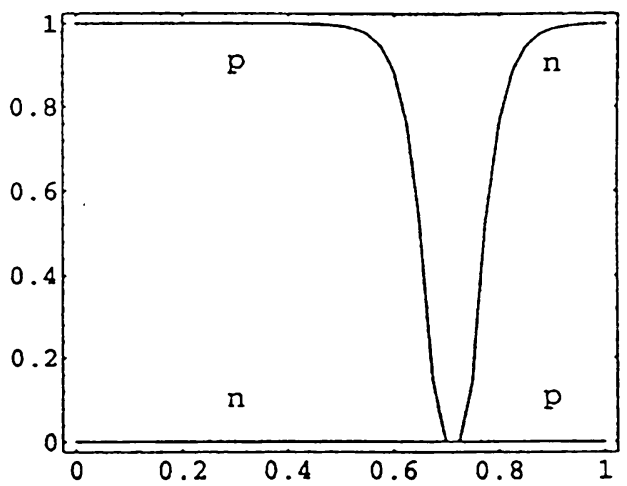

(d)

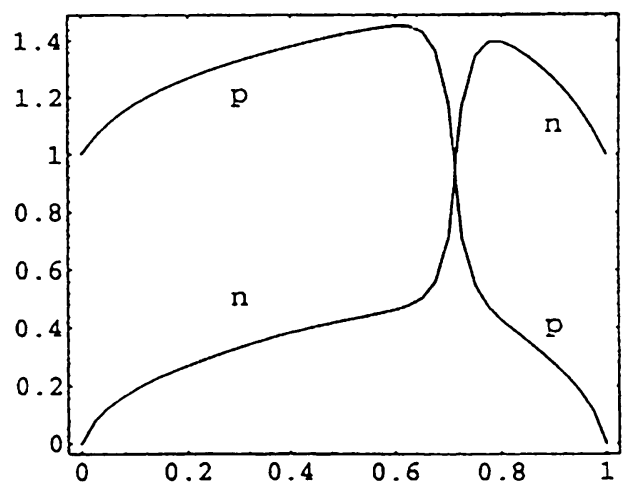

FIG. 1. Carrier densities (in $10^{15} \mathrm{~cm}^{-1}$ ) of a $p n$-diode vs. position (in $10^{-3} \mathrm{~cm}$ ) for a) thermal equilibrium, b) $U<0$, c) $0<U<U_{\mathrm{th}}$, d) $U>U_{\mathrm{th}}$.

(4.1)-(4.3), (4.5), (4.6) can now be written as

$$
\begin{gathered}
\lambda^{2} V^{\prime \prime}=g\left(V-\varphi_{n}+h\left(n_{i}\right)\right)-g\left(-V+h\left(n_{i}\right)+U\right)-C, \\
-g\left(V-\varphi_{n}+h\left(n_{i}\right)\right) \varphi_{n}^{\prime}=J_{n}, \quad J_{n}^{\prime}=0, \\
V(0)=h(C(0))-h\left(n_{i}\right), \quad V(1)=h\left(n_{i}\right)-h(-C(1))+U, \\
\varphi_{n}(0)=0, \quad \varphi_{n}(1)=U-h(-C(1))+2 h\left(n_{i}\right) .
\end{gathered}
$$

As the next step we introduce a small parameter $\delta>0$ and set

$$
U=U_{\mathrm{th}}+\delta u, \quad u>0 .
$$

Denoting by $V_{\mathrm{th}}$ the solution of (4.12) with $U=U_{\text {th }}$ we rescale the unknowns by

$$
V=V_{\mathrm{th}}+\delta v, \quad \varphi_{n}=\delta \varphi, \quad J_{n}=\delta^{\alpha} j_{n}
$$


We shall carry out the formal limit $\delta \rightarrow 0$ in the problem for $V, \varphi$, and $j_{n}$. Substituting (4.15), (4.16) in (4.14) and letting $\delta \rightarrow 0$ in the first and third lines, we obtain the limiting problem for $v$ :

$$
\begin{gathered}
\lambda^{2} v^{\prime \prime}=\left[g^{\prime}\left(V_{\mathrm{th}}+h\left(n_{i}\right)\right)+g^{\prime}\left(-V_{\mathrm{th}}+h\left(n_{i}\right)+U_{\mathrm{th}}\right)\right] v, \\
v(0)=0, \quad v(1)=u,
\end{gathered}
$$

having a unique solution since the coefficient on the right-hand side of the differential equation is positive.

The rescaled current relation and the boundary conditions for $\varphi$ read

$$
\begin{gathered}
-g\left(V_{\mathrm{th}}+h\left(n_{i}\right)+\delta(v-\varphi)\right) \varphi^{\prime}=\delta^{\alpha-1} j_{n} \\
\varphi(0)=0, \quad \varphi(1)=u .
\end{gathered}
$$

In the limit the coefficient of $\varphi^{\prime}$ is positive in the interior of the interval and vanishes at $x=1$. Because of $\alpha>1$ we expect the limit of $\varphi$ to be constant and equal to its value 0 at $x=0$ in $[0,1)$. Near $x=1$, we construct a boundary layer. In terms of the stretched variable $\xi=\frac{1-x}{\delta}$, the argument of $g$ in (4.17) is $O(\delta)$. Omitting $O\left(\delta^{2}\right)$-terms we obtain

$$
g\left(\delta\left(v_{0} \xi+u-\varphi\right)\right) \dot{\varphi}=\delta^{\alpha} j_{n}
$$

with the dot denoting derivation with respect to $\xi$ and $v_{0}>0$ being determined from $V_{\mathrm{th}}=-h\left(n_{i}\right)+v_{0}(1-x)+O\left((1-x)^{2}\right)$ as $x \rightarrow 1$. A simple computation using the pressure law (2.4) shows that $g$ is homogeneous of degree $\alpha$, and therefore the above equation is equivalent to

$$
g\left(v_{0} \xi+u-\varphi\right) \dot{\varphi}=j_{n}
$$

It has to be solved for $\xi \in[0, \infty)$ and the constant $j_{n}<0$ to be determined such that the boundary conditions $\varphi=u$ for $\xi=0$ and $\varphi=0$ for $\xi=\infty$ are satisfied. The solution of the above equation subject to the boundary condition at $\xi=0$ is given implicitly by

$$
\int_{0}^{v_{0} \xi+u-\varphi} \frac{g(z) d z}{v_{0} g(z)-j_{n}}=\xi
$$

which can be rewritten as

$$
\int_{0}^{v_{0} \xi} \frac{j_{n} d z}{v_{0}\left[v_{0} g(z)-j_{n}\right]}+\int_{v_{0} \xi}^{v_{0} \xi+u-\varphi} \frac{g(z) d z}{v_{0} g(z)-j_{n}}=0
$$

Because of the properties of $g$ we can pass to the limit $\xi \rightarrow \infty$ in both integrals in the above equation. As the result we obtain the voltage-current characteristic, which can be 
written as

$$
j_{n}=-v_{0}\left(\frac{u}{\kappa}\right)^{\alpha}
$$

or, in terms of our original variables,

$$
J_{n}=-v_{0}\left(\frac{U-U_{\mathrm{th}}}{\kappa}\right)^{\frac{1}{\gamma-1}} \quad \text { as } U \rightarrow U_{\mathrm{th}}+
$$

with

$$
\kappa=\int_{0}^{\infty} \frac{d z}{g(z)+1}
$$

We conclude with a short discussion of the case of nonvanishing recombinationgeneration effects. Consider problem (4.1), (4.2), (4.3), (4.5), (4.6) with the continuity equations (4.2) replaced by

$$
J_{n}^{\prime}=-J_{p}^{\prime}=R
$$

where $R$ satisfies (2.16), (2.17). The proof of Theorem 3.1 shows that the potential satisfies the semilinear elliptic equation (compare to (3.3))

$$
\lambda^{2} V^{\prime \prime}=g\left(V-\varphi_{n}+h\left(n_{i}\right)\right)-g\left(\varphi_{p}-V+h\left(n_{i}\right)\right)-C .
$$

Using the $L^{\infty}$-estimates for the quasi-Fermi potentials, comparison function arguments show that for small applied voltages $V, \varphi_{n}$ and $\varphi_{p}$ are small $L^{\infty}$-perturbations of their equilibrium versions $V=V_{e}, \varphi_{n}=\varphi_{p}=0$. Therefore, since vacuum regions for both electrons and holes occur at thermal equilibrium, it is obvious from the relations $n=$ $g\left(V-\varphi_{n}+h\left(n_{i}\right)\right), p=g\left(\varphi_{p}-V+h\left(n_{i}\right)\right)$ that the same is true for small enough applied voltages.

For vanishing recombination-generation the electron and hole current densities are constant and, thus, the occurrence of vacuum regions for both types of charge carriers implies that the total current vanishes. This cannot be expected if recombination-generation effects are accounted for. In this case the following scenario is most likely: away from thermal equilibrium the current is nonzero. In the hole vacuum region it is a pure electron current and a pure hole current in the electron vacuum region. In a layer around the $p n$-junction, where both carrier densities are positive, recombination-generation effects are responsible for the electron current gradually being taken over by the holes or vice versa.

These speculations are confirmed by the results of numerical computations. Figure 2 shows numerically computed voltage-current characteristics for vanishing and nonvanishing recombination-generation rates. In the latter case the model

$$
R(n, p)=\left(n+\left(n_{\mathrm{vac}}-p\right)^{+}\right)\left(p+\left(n_{\mathrm{vac}}-n\right)^{+}\right)\left(h(n)+h(p)-2 h\left(n_{i}\right)\right),
$$

satisfying $(2.16),(2.17)$ was used in the computations. 
a)

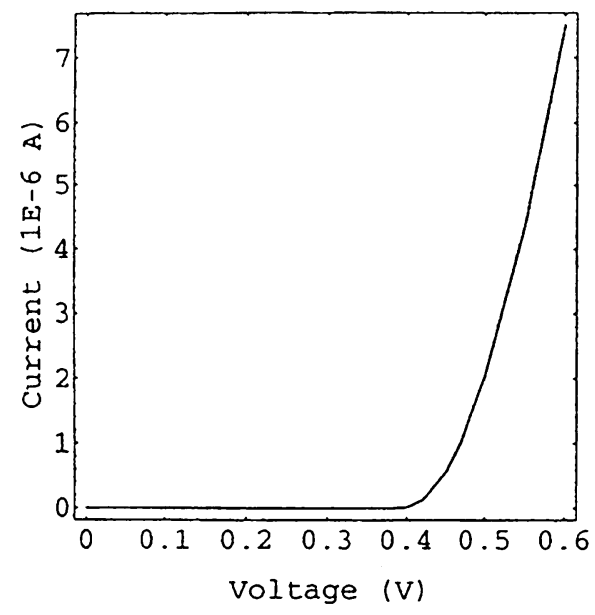

b)

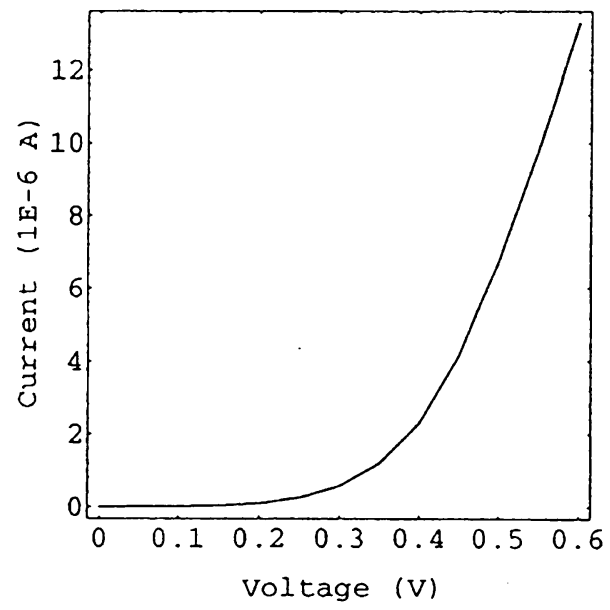

FIG. 2. Voltage-current characteristics of a $p n$-diode for a) $R=0$, b) $R \neq 0$.

Acknowledgment. The work of the first author has been supported by the ECnetwork, contract \#ERBCHRX-CT930-413 and by the Deutsche Forschungsgemeinschaft, grant numbers MA 1662/-1 and -2 . The work of the second author has been supported by the Austrian Fonds zur Förderung der wissenschaftlichen Forschung under grant number P08571-PHY.

\section{REFERENCES}

[1] A. Jüngel, The free boundary problem of a semiconductor in thermal equilibrium, Math. Meth. in the Appl. Sci. 18, 387-412 (1995)

[2] A. Jüngel, Numerical approximation of a drift-diffusion model for semi-conductors with nonlinear diffusion, ZAMM 75, 783-799 (1995)

[3] P. A. Markowich, C. Ringhofer, and C. Schmeiser, Semiconductor Equations, Springer-Verlag, Wien, 1990

[4] P. A. Markowich and A. Unterreiter, Vacuum solutions of a stationary drift-diffusion model, Ann. d. Scuola Normale Sup. Pisa 20, 371-386 (1993)

[5] A. Unterreiter, Vacuum and non-vacuum solutions of the quasi-hydrodynamic semiconductor model in thermal equilibrium, Math. Meth. in the Appl. Sci. 18, 225-254 (1995) 